\title{
Woman with bilateral painful lower limb swelling: Case report of an atypical presentation of toxoplasmosis in immunocompetent adult
}

Verni $\mathrm{S}^{1}$, Perera $\mathrm{KJC}^{2}$, Paramanantham $\mathrm{A}^{2}$, MunasingheTMJ ${ }^{3}$

\section{Summary}

A 43 year old previously healthy woman presented with painful bilateral lower limb swelling for 3 weeks, and fever and polyarthralgia of 3 days duration. She had a history of close contact with cats. Examination revealed moderate hepatomegaly with severe muscle tenderness. She had eosinophilia and elevated creatinine kinase and lactate dehydrogenase. Results of her toxoplasmosis serology were consistent with acute toxoplasmosis. Immunosuppressive conditions were excluded. Bone marrow biopsy revealed marked eosinophilia. According to these findings she was diagnosed as having acute toxoplasmosis complicated with myositis. This was further strengthened by her response to pyrimethamine and sulfadiazine treatment.

\section{Background}

Toxoplasmosis is a worldwide zoonosis. It is caused by Toxoplasma gondii, an intracellular protozoan parasite. Though $95 \%$ of some population are infested (1), it is uncommon to cause clinical disease. $80-90 \%$ of immunocompetent patients are asymptomatic during acute toxoplasmosis and only $10-20 \%$ of adults and children are symptomatic (2). The patient develops a flu-like illness characterised by fever, lymphadenopathy, malaise, myalgia and maculopapular rash that spares the palms and the soles. Hepatosplenomegaly also can be present. The most common manifestation in immunocompetent individuals is lymphadenopathy. After acute infection, only a small percentage will go on to develop chorioretinitis, lymphadenitis and even more rarely myocarditis and polymyositis.

Myositis is a rare clinical presentation in an immunocompetent adult with toxoplasmosis (2). Here we present a case of an immunocompetent lady with atypical presentation of toxoplasmosis with myositis.

\section{Case Presentation}

A 43 year old previously healthy woman from the western province presented with a history of painful bilateral lower limb swelling of 3 weeks, along with fever and painful multiple large joints in the lower limbs of 3 days duration. She had a history of close contact with cats. There was no history suggestive of connective tissue disease, allergic, thrombotic episodes or recent travel.

The patient was febrile and had bilateral lower limb swelling up to knees with severe muscle tenderness. Moderate hepatomegaly was present without splenomegaly or lymphadenopathy. There were no skin lesions and joint examination was normal other than mild tenderness in large joints. The neurological and fundal examinations were normal.

\section{Investigations and management}

The haemoglobin was $10.2 \mathrm{~g} / \mathrm{dl}$; eosinophil count was $4.514 \times 10^{9} / \mathrm{l}$ and she had a platelet count of $328 \times$ $10^{9} /$ l. Blood picture showed marked eosinophilia with mild rouleaux formation. The CRP was 138 and ESR was $20 \mathrm{~mm} / 1^{\text {st }}$ hour. The LDH was $823 \mathrm{U} / \mathrm{l}$. She also had an elevated CPK, $1695 \mathrm{U} / \mathrm{l}$. ALT and AST were 195U/l and $106 \mathrm{U} / \mathrm{l}$ respectively and serum albumin was $25 \mathrm{~g} / \mathrm{l}$.

The bone marrow biopsy showed a reactive marrow with marked eosinophilia. Filarial antigen was negative. Renal function, electrocardiogram, 2D transthoracic echocardiogram, stool full report including parasite analysis , serum for ANA and rheumatoid factor, doppler scan of lower limb, and NCCT brain were normal. Toxoplasma IgM was reactive and IgG was positive with titre more than 150. Toxocara antibody, both IgM and IgG were negative.

Based on the clinical history, haematological findings and serologic assay, it was concluded that she had acute toxoplasmosis. Immunosuppressive conditions were ruled out as the fasting blood sugar and retroviral screening were negative. Unfortunately the patient was not willing for muscle biopsy. We started treatment with pyrimethamine $100 \mathrm{mg}$ loading dose followed by $50 \mathrm{mg}$ daily and sulfadiazine $500 \mathrm{mg} 6$ hourly with folic acid $5 \mathrm{mg}$ 
twice daily for six weeks. Her symptoms resolved and both full blood count and eosinophil count became normal along with her LDH and CPK. At 3 months of follow up, liver was not palpable and liver enzymes were normal. IgM was non-reactive but the IgG remained positive.

\section{Discussion}

Toxoplasmosis is higher in incidence in regions that have hot, humid climates and lower altitudes. Cats are essential in the lifecycle of Toxoplasma gondii because they are the only hosts that can excrete the environmentally resistant oocysts. Infection in humans occur most commonly through the ingestion of raw or undercooked meat that contains tissue cysts, through water or food contaminated with oocysts, or congenitally through transplacental transmission from a mother who acquired the infection during gestation. Initial infection and resultant seeding of different organs, is followed by chronic or latent infection by tissue cyst without any significant clinical manifestations. Most common sites of latent infection are brain, eye, heart, skeletal muscle, smooth muscle and cardiac muscle.

Laboratory studies are vital to establish the diagnosis as the clinical manifestations of the infection are variable. Myositis resembling polymyositis as a manifestation of acute toxoplasmosis in immunocompetent patients has been reported infrequently when compared to immunodeficient patients $(3,4)$. Our patient had muscle pain with high creatinine kinase and lactate dehydrogenase levels, hepatomegaly, marked eosinophilia, history of exposure to cats and response to treatment with pyrimethamine and sulfadiazine, all of which when taken collectively are suggestive of a diagnosis of myositis following toxoplasmosis in an immunocompetent patient.

\section{Learning points}

We suggest that toxoplasmosis should be considered as a differential diagnosis even in immunocompetent patients who have features of myositis, arthralgia, and hepatomegaly and marked eosinophilia with elevated muscle enzymes.

\section{Abbreviations}

ALT= Alanine transaminase, ANA= Antinuclear antibody, $\mathrm{AST}=$ Aspartate transaminase,

$\mathrm{CPK}=$ creatinine kinase, $\mathrm{CRP}=\mathrm{C}$ - reactive protein, $\mathrm{ESR}=$ erythrocyte sedimentation rate, $\mathrm{LDH}=$ Lactate dehydrogenase, NCCT = Noncontrast computed tomography

\section{References}

1. MadhavaGunaseekera, Weekly Epidemiological Report, 2013, 8- 14 June; Volume 40, No 24, Epidemiology Unit, Ministry of Health, Sri Lanka

2. Mandell GL, Bennet JE, Dolin R. Mandell, Douglas, and Bennett's principles and practices of infectious disease. 2010; 7th edition; Volume 2: 3495. ISBN 978-0-4430-6839-3

3. Montoya JG, JordanR, Lingamneni S, et al. Toxoplasmic myocarditis and polymyositis in patient with acute acquired toxoplasmosis diagnosed during life. Clinical Infectious Diseases. 1997;24 (4):676-683. Available from: http://www.jstor.org/stable/4481073

4. Paspalaki PK, Mihalidou EP, Bitsori M, Tsagkaraki D, Mantzouranis E. Polymyositis and myocarditis associated with acquired toxoplasmosis in immunocompetent girl. BMC Musculoskeletal Disorder. 2001; 2:8: doi: 10.1186/1471-2474-2-8. 